\title{
Strategies and Steps for the Accomplishment of Bioequivalence Regulations in Chile
}

\author{
Valenzuela $\mathrm{MT}^{1}$ and Aceituno $\mathrm{A}^{\mathbf{2}^{*}}$ \\ ${ }^{1}$ University of Los Andes, Chile \\ ${ }^{2}$ Facultad de Farmacia, Universidad de Valparaíso, Chile
}

\begin{abstract}
The process of bioequivalence policy implementation in Chile is presented. A chronological description of the regulatory bodies and tools to help fulfilling this legal requirement as well as the methodology to select drugs and their drug products subject to demonstrate bioequivalence is presented. Also, it is depicted the strategies used by health authorities to implement this new drug policy in order to not affecting drug product availability, increasing generics penetration in the pharmaceutical market and improving access to medicines both in the public and private healthcare system as well. Overall, it is expected that the implementation of the bioequivalence policy would contribute to health expenditure containment allowing redirecting the health budget to other sanitary needs as the most important challenge to come.
\end{abstract}

\section{Keywords: Bioequivalence; Generics; Process validation; Drug policy}

Abbreviations: BE: Bioequivalence; MSDP: Multisource Drug Product; RP: Reference Product; WHO: World Health Organization; ANAMED: Agencia Nacional de Medicamentos; ISP: Instituto de Salud Pública; CRO: Contract Research Organization; FDA: Food and Drug Administration; EMA: European Medicine Agency; ANVISA: Agencia Nacional de Vigilancia Sanitaria; GMP: Good Manufacturing Practices, NTI: Narrow Therapeutic Window; GES: Garantías Explícitas en Salud.

\section{Introduction}

Since the publication of the bioequivalence policy in 2005, the Chilean Regulatory Agency, i.e. Institute of Salud Pública of Chile, has made mandatory that listed drugs and their drug products required to demonstrate bioequivalence to do so [1]. Bioequivalence (BE) is a method with straightforward scientific bases where the fundamental principle is that the systemic effect of a multisource pharmaceutical product (MSDP) will be near the same as the pharmacological effect produced by a reference pharmaceutical product (RP) [2]. The RP is the one selected by the Regulatory Agency based on its evidence of safety and efficacy and it can be subsequently employed by generic companies to perform comparative pharmacokinetics studies [3]. The near similar pharmacological effect of a generic product is not only consequence of the similar amount of active ingredient contained in the unitary dosage form when compared to the RP but also of the overlapping drug plasma concentration profiles resulting from either the reference or generic product oral administration [4]. As such, the assessment of bioequivalence is a regulatory requirement for any generic product to scientifically sustain the efficacy and safety of a MSDP when compared to the RP. The World Health Organization (WHO) encourages that this regulatory requirement be applied in developing countries and that the local regulatory authorities issue norms, decrees and guidance documents to help regulated companies to comply with this requisite [5]. The need of bioequivalence testing to support drug safety and efficacy has become a standard requisite in developed countries in order to increase health cost saving and improved patient access to safe and effective medicines [6]. This article describes the process of the bioequivalence policy implementation in Chile, the strategies used to accomplish this new drug regulatory burden and the sanitary effects expected on the pharmaceutical market and healthcare system.

\section{Drug regulatory framework}

In Chile drug regulations rely on a legal basis namely the Sanitary Code, The Supreme Decree \#3 and several technical guidelines that helped supporting the implementation of bioequivalence requirements. The Sanitary Code in its $94^{\text {th }}$ article defines the entity with overall responsibility on medicine quality in the whole national territory as well as with the compliance of dispositions described in the Code and its regulations. Supreme Decree \# 3 established the creation of ANAMED (i.e. National Medicines Agency) and its Bio-pharmaceutics and Bioequivalence division to be in charged of looking after the compliance of bioequivalence and process validation requirements $[7,8]$. Although the country had the afore mentioned regulatory framework since 2004 it was only during the year 2009 that the government launched the first Exempt Decree describing listed drugs and dosage forms that should comply with bioequivalence assessment. Lately in 2011, The Public Health Institute of Chile (ISP) divulged the technical criteria to prioritize which additional drugs in conventional dosage forms would be advisable to demonstrate bioequivalence and further Decrees were launched.

At the beginning the policy of bioequivalence implementation was not free from hurdles as the number of centres or contract research organizations (CROs) where to perform the studies were insufficient in number. Therefore, the ISP invited Universities with health career fields to add up in the effort to create CROs as these institutions had highly qualify personnel and also they were willing to implement facilities where to carry out the bioequivalence assays. The selection of a reference product and the lack of experience to present properly bioequivalence study dossiers were additional obstacles along with the resistance to change produced by these regulations. Therefore, the regulatory agency created the Bio-pharmaceutics and Bioequivalence division as part of the drug regulatory department called ANAMED, a unit being composed of highly qualified professionals trained in technical aspects such manufacturing process validation, evaluation of bioequivalence dossiers, reference product selection and certification/ audit of national and international BE centres.

*Corresponding author: Aceituno A., PhD. Facultad de Farmacia, Universidad de Valparaíso, Chile, E-mail: aaceituno@ispch.cl

Received June 12, 2018; Accepted June 26, 2018; Published July 01, 2018

Citation: Valenzuela MT, Aceituno A (2018) Strategies and Steps for the Accomplishment of Bioequivalence Regulations in Chile. Pharmaceut Reg Affairs 7: 204. doi: 10.4172/2167-7689.1000204

Copyright: ( 2018 Valenzuela MT, et al. This is an open-access article distributed under the terms of the Creative Commons Attribution License, which permits unrestricted use, distribution, and reproduction in any medium, provided the original author and source are credited. 


\section{Implementing bioequivalence policy}

All drug product that had already been registered as similar to a previously registered innovator product and they were commercialized without bioequivalence testing was given a deadline to do so. These pharmaceutical products were required to demonstrate $\mathrm{BE}$ by performing in vitro or in vivo studies in certified $\mathrm{BE}$ centres. BE centres were either certified and authorized by the ISP itself ( 7 centres in Chile) or they were recognized by the Chilean regulatory agency as long as the centre was audited under the jurisdiction of foreign regulatory agencies known to be as highly sanitary surveillance agencies such as FDA, EMA, ANVISA and some others. Besides, the pharmaceutical good should demonstrate the compliance with cGMP and process validation with three industrial batches of the drug product. If the pharmaceutical company had designed and formulated the drug product under the concept of a controlled drug product development process, one batch (exhibit batch) was required to accept bioequivalence testing and a time line was given to the company to complete the validation process with 2 or more additional batches. Retrospective and prospective validations were also acceptable along with concurrent validation process [9].

\section{Stepwise procedure to implement bioequivalence requirements}

Once the normative and technical guidelines were completed and ready to be launched and applied, a prioritized selection of drugs and their drug products was performed to comply with bioequivalence assessment and a sanitary criteria was followed in order to avoid drug product shortcuts due to this regulatory burden namely sanitary risk, narrow therapeutic index (NTI)drugs, chronic disease treatments, explicit guarantees healthcare program (GES), dosage form types (starting with conventional oral solid dosage forms), health care expenditures, availability of the RP and number of generic versions in the pharmaceutical market (branded generics and INN). This criteria was applied to MSDPs seeking registration as well as the ones already authorized and commercialized without BE testing.

\section{Drug Products Seeking Registration (Abbreviate New Drug Applications)}

Currently abbreviate new drug applications are required to demonstrate BE by performing comparative in vitro or in vivo studies in certified centres if the drug product contains one of the drugs listed in any of previous exempt decrees launched. The Chilean Agency has authorized eleven in vitro biopharmaceutical centres, most of them being part of the quality control sections of pharmaceutical companies. These biopharmaceutical centres are able to demonstrate bioequivalence by performing comparative dissolution profiles and drug solubility analysis [10]. In addition pharmaceutical companies are also required to demonstrate compliance with cGMP and manufacturing process validation. In contrast to manufacturing process validation requirements for already registered products, validation for products seeking registration can be demonstrated with a pilot size batch or by implementing a robust formulation process; the bioequivalence study results may be acceptable under these conditions. The pharmaceutical company must however submit a timeline scheme indicating the period needed to complete the manufacturing process validation on industrial size batches (usually 3) [9].

\section{Bio-waivers}

In vitro methods are based on the BCS drug classification approach [11]. This approach allows granting bio- waivers to class 1 and 3 drugs formulated in conventional rapid or very rapidly dissolution dosage forms. Exceptionally, class 2 drugs were accepted as candidates for bio-waivers in the pass but currently this is no longer permitted as per WHO recommendations [12]. In vitro studies can be also used to demonstrate $\mathrm{BE}$ of higher or lower dose strengths of a drug product whose $\mathrm{BE}$ has been demonstrated by in vivo methods for one dose strength (biobatch). This procedure is known as dose strength based bio-waivers, and in contrast to the BCS approach, similarity between lower o higher dose strengths with the biobatch can be assessed by comparison of dissolution profiles in a discriminative dissolution medium $[12,13]$.

\section{Bioequivalence testing by in vivo approaches}

In vivo studies are performed following compliance with good clinical practices (GCP) in healthy volunteers in authorized centres. Usually, the centres may perform all the steps involved, i.e. clinical, analytical and statistical/pharmacokinetic phases. In other situations, agreements among institutions running one or two phases are also acceptable, as long as all of them have been previously authorized by the Chilean National Agency. In order to avoid failures due to a poor study design, the Chilean National Agency must authorize the study protocols before the submission of the final study report. To be approved, the study protocol must include the following information: analytical method validation, information about the drug inter and intra-subject variability, proposed numbers of volunteers, sequenceperiod design, statistical potency expected, ethics committee approval, signed written consent etc. [14].

One challenge that the National Agency had to address was that of selecting the appropriate reference drug product. This was the case for MSDPs whose innovator product had been discontinued worldwide. Under these circumstances, the regulation established that a local product must be selected as the reference product. A decision tree was followed in order to select the best drug product candidate among the local alternatives (Figure 1). Criteria such as validation process compliance, pharmacovigilance programs and maintenance of quality standards post $\mathrm{BE}$ certifications were required in order to being selected as the reference product.

\section{Decrees Launched by the Sanitary Authority, Therapeutic Targets and Number of Drug Products to be Certified as Therapeutic Equivalents}

The first Decree launched by the Ministry of Health was the Exempt Decree \# 500. This one describes the drugs, the clinical uses and the number of MSDPs subject to the requirement. This original decree has been modified several times to increase the number of drug products under the $\mathrm{BE}$ requirement (Figure 2) and expanding the requirement for non-conventional dosage forms (extended release). In terms of number of drugs, until now 189 active substances have been selected as required to demonstrate $\mathrm{BE}$ in conventional or non-conventional dosage forms. Described in Figure 3 was the main criteria to select drugs and their drug products subject to demonstrate bioequivalence; this strategy was followed in order to prioritize human and technical capabilities and to weigh up the sanitary impact of this regulatory burden. Shown in Figure 4 is the progression in the number of drug products that has been proved to be bioequivalent to the reference product and can be interchangeable since 2010. With the aim of identifying what pharmaceutical products have proved to be bioequivalent to the reference products, a logo was designed and approved. The logo goes onto the secondary packaging of drug products whose bioequivalence has been certified and it is a transitory step necessary for patients to distinguish whether their selected drug products are bioequivalent or not (Figure 4). From a universe of 6000 


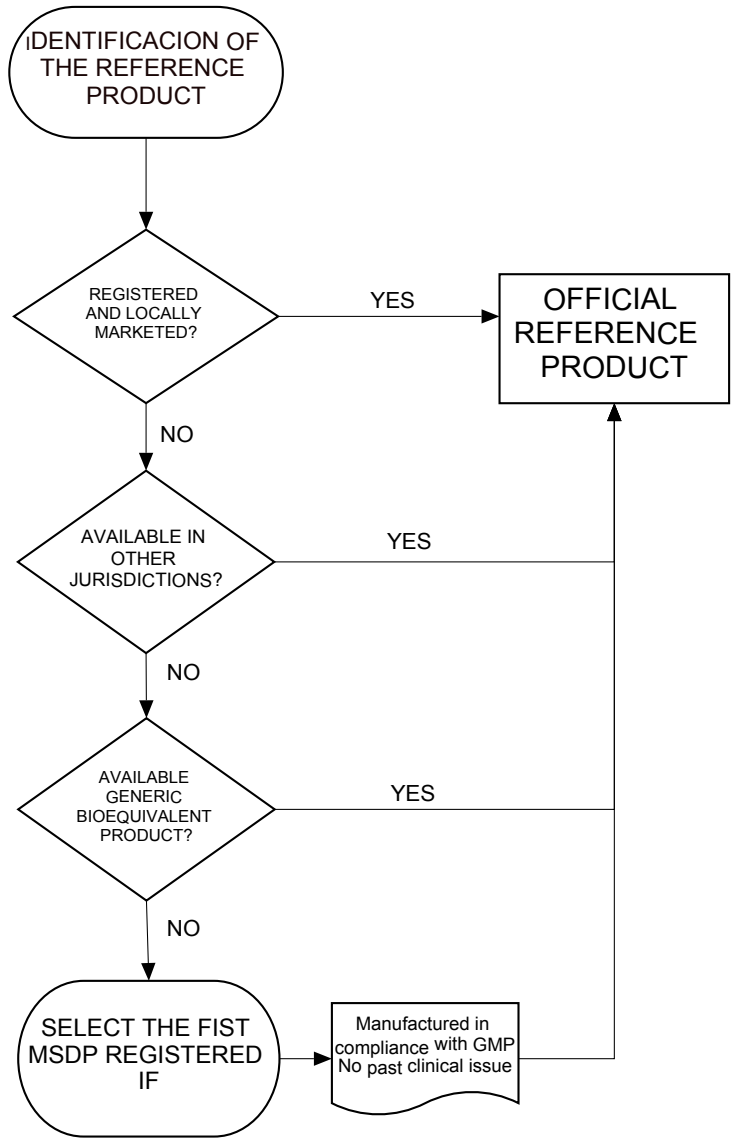

Figure 1: Decision tree to select the RP to be used in bioequivalence assays.

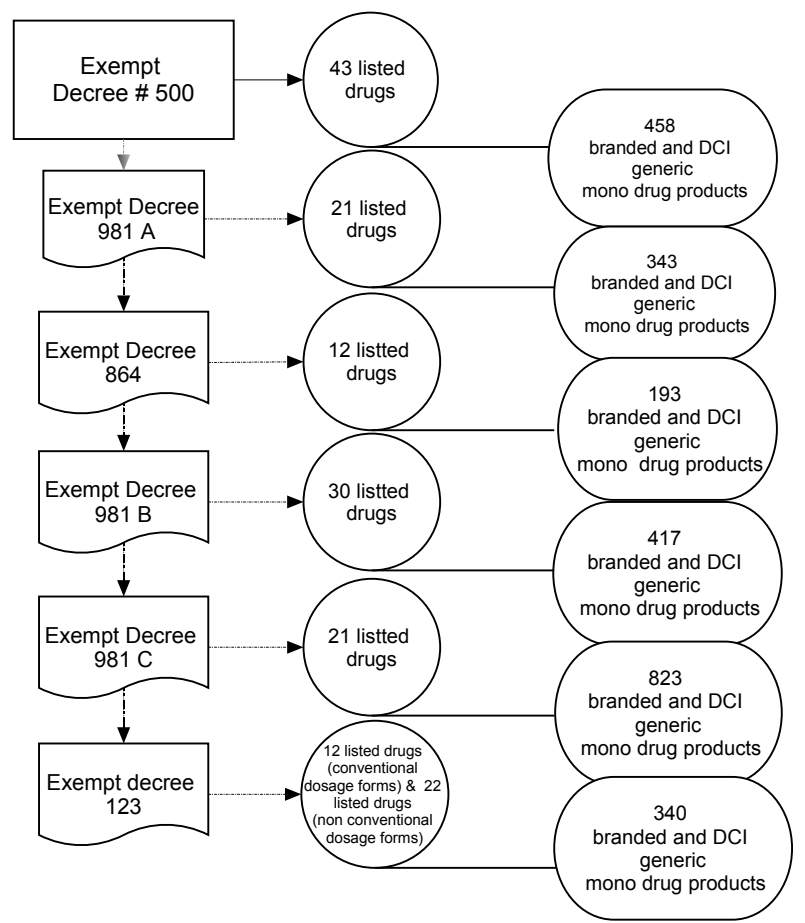

Figure 2: Exempt decrees that listed selected drugs and their number of drug products ( $\mathrm{DCl}$ and branded generics) subject to demonstrate bioequivalence.

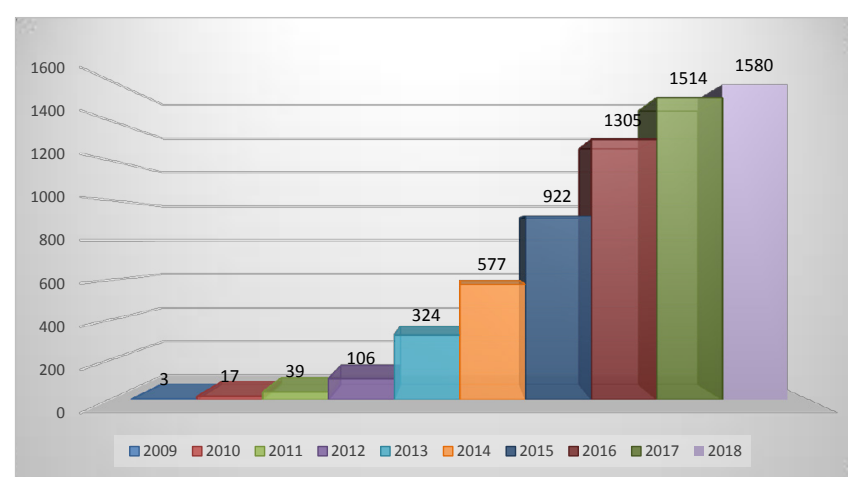

Figure 3: Approved drug products with therapeutic equivalence evaluations (2009-2018).

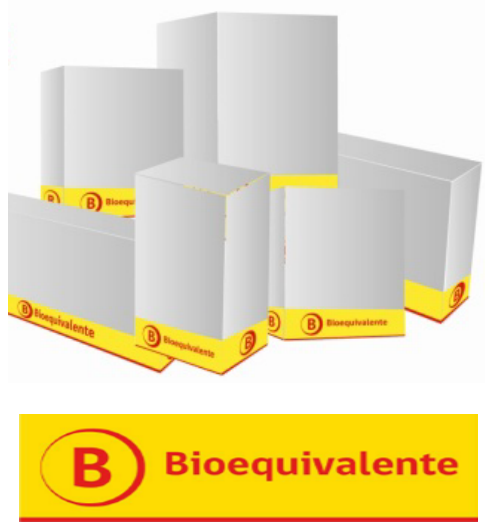

Figure 4: Logo currently used to identify drug products certified as bioequivalent in the Chilean pharmaceutical market.

MSDPs subject to demonstrate bioequivalence, about 1600 drug products have proved to be bioequivalence and interchangeable with the reference products and therefore they are allowed to be labelled as such (Figure 3 ). The logo would not be necessary once all drug products either branded or INN generics have proved to be bioequivalent to their respective reference products.

The generic medicine policy has allowed a patient centred approach as it is believed to represent equal chances of access to a safe and effective therapeutic treatment no matter the source of the drug products chosen In addition pharmaceutical companies 'benefits from the bioequivalence policy by improving and strengthening their GMP standards compliance and manufacturing process validation. The impact of generics penetration on healthcare systems has been recognized due to the significant decrease of healthcare expenditures. The high cost of medications in our country means that families spend close to $40 \%$ of their total monthly health budget on medicines only. Although at the beginning the goal was focused on improving people access to cheaper drug products, it was realized later that this did not depend on actions taken by the State but on the willingness of the pharmaceutical industry to manufacture INN products instead of branded generics. Branded generics are sometimes as expensive as the brand name original product so this medicine policy effect on drug product prices was marginal. Overall, the generics (branded or not) still produce health costs containment compared to the innovators but this effect is not seen when the pharmaceutical market is composed mostly of branded kind MSDPs. 
Currently, the process of bioequivalence implementation in Chile has slowed down due to multiple reasons: on the health authority side, the fear to produce drug shortcuts as a consequence of this regulatory burden, pharmaceutical pressure to postpone the original deadlines, changings in healthcare priorities and some other reasons. By all means, we believe the state policy thinking is a future oriented matter with solid bases and bioequivalence assessment has arrived to stay.

\section{Final Remarks}

Bioequivalence testing is an increasingly important requisite to sustain drug product efficacy of generic versions of the innovator products. In addition, this new regulation has allowed that pharmaceutical companies raise their standards in terms of GMP compliance. People benefit from having a standard quality among different generic versions of the same innovator (same efficacy) at lower costs in a country where healthcare expenditure heavily relies on the family budget. The challenge is now to increase the number of BE certified medicines and expand the regulatory requisite to dosage forms other than solid oral dosage forms as it is estimated that only $25 \%$ of MSDPs has proved to be bioequivalent.

\section{References}

1. https://www.leychile.cl/Navegar?idNorma $=1036802$

2. VD Koralis (2016) From Bioequivalence to Bio-similarity: The Rise of a Nove Regulatory Framework. Drug Res (Stuttg) 66: 1-6.

3. David B, Braddy AC, Conner DP, Yu LX (2013) International International Guidelines For Bioequivalence of Systemically Available Orally Administered Generic Drug Products: A Survey of Similarities and Differences. The AAPS J 4: $974-990$
4. Zhang X, Zheng N, Lionberger A, Yu LX (2013) Innovative Approaches for Demonstration of Bioequivalence: the US FDA Perspective. Ther Deliv 4: 725-740.

5. Cristancho RA, Andia T, Barbosa T, Watanabe J (2015) Definition and classificacion of Generic Drugs Across the World. Appl Health Econ Health Policy 13: S5-S11.

6. Hassali MA, Alrasheedy AA, McLachlan A, Nguyen TA, AL-Tamimi, et al (2014) The Experiences of Implementing Generic Medicine Policy in Eight Countries: A Review and Recommendations for a Successful Promotion of Generic Medicine Use. Saudi Pharm J 22: 491-503.

7. https://www.leychile.cl/Navegar?idNorma=5595

8. http://www.ispch.cl/sites/default/files/decreto_3_0.pdf

9. World Health Organization (2006) WHO Expert Committee on Specifications for Pharmaceutical Preparations, Fortieth Report, Annex 7: Multisource (generic) pharmaceutical products guidelines on registration requirements to establish interchangeability, WHO Technical Report Series N937, Geneva.

10. Lennernas H, Abrahamsson B (2005) The Use of Biopharmaceutic Classification of Drugs in Drug Discovery and Development: Current Status and Future Extension. J Pharm Pharmacol 57: 273-285.

11. Shekhawat PB, Pokharkar V (2017) Understanding Peroral Absorption: Regulatory Aspects and Contemportay Approaches to Tackling Solubility and Permeability Hurdles. Acta pharm Sin B 7: 260-280.

12. Verbeeck RK, Musuamba FT (2012) The revised 2010 EMA guidelines for the investigation of Bioequivalence for immediate release oral formulations with systemic action. J Pharm Pharmaceut Sci 15: 376-388.

13. Machado JC, Lange V, Todeschini V, Volpato NM (2014) Development and validation of discriminative dissolution method for atorvastatin calcium tablets using in vivo data by LC and UV methods. AAPS Pharm SciTech 15: 189-197.

14. Establish technical guides for conducting studies of bioequivalence of monodroga pharmaceutical products of conventional release and forms for submitting the required background for the realization of such studies. 